\title{
Modelling Microstructure and Its Effects during Multipass Hot Rolling
}

\author{
John H. BEYNON and C. Michael SELLARS ${ }^{1)}$ \\ Department of Engineering, The University, Leicester LE1 7RH, U. K. \\ S1 3JD, U.K. \\ 1) School of Materials, The University, Sheffield
}

(Received on September 17, 1991; accepted in final form on November 15, 1991)

\begin{abstract}
By collaborative work the Sheffield Leicester Integrated Model for Microstructural Evolution in Rolling (SLIMMER) has been developed for hot rolling of flat products. The background physical metallurgy is presented together with the expressions used to describe microstructure evolution for a range of ferrous and non-ferrous metals. The finite difference thermal model at the heart of SLIMMER computes heat loss to air, descalers, rolls and water cooling while allowing for oxidation and deformation heating. The use of temperature compensated time enables isothermally determined equations for microstructure evolution to be applied to practical non-isothermal conditions. Rolling loads and torques are calculated using Sims theory with an accurate prediction of mean flow stress. Examples of rolling niobium microalloyed steel plate and the effect of initial grain size illustrate the capabilities of SLIMMER and show some of the validation of the predictions.
\end{abstract}

KEY WORDS: microstructure; modelling; thermomechanical processing; recrystallization; precipitation; grain growth; rolling; aluminium; steel; SLIMMER.

\section{Introduction}

The basis for current understanding of the physical metallurgy of hot working was developed in the $1960 \mathrm{~s}^{1,2)}$ This work demonstrated the high degree of sensitivity of microstructure to the operating conditions. The determining variables are normally characterized by temperature $(T)$, strain $(\varepsilon)$ and strain rate $(\dot{\varepsilon})$, with flow stress $(\sigma)$ being the measurable response of the microstructure to these conditions. Having quantified the kinetics and outcome of microstructural events in terms of temperature and the deformation, applying this knowledge to an industrial process requires that the thermomechanical conditions are themselves well defined.

The first priority for a process model should be temperature since it has a dominant influence on the microstructure. Hollander ${ }^{3)}$ produced the first comprehensive temperature model for the hot rolling of flat products using a finite difference technique. Harding ${ }^{4)}$ later produced a more detailed solution which forms the basis of the finite difference model to be described below. Modelling the thermal and mechanical conditions of the flat rolling process has since become a popular activity, exemplified by the appearance of a text on the subject. ${ }^{53}$ However, for following the evolution of microstructure down a rolling mill, the formulae describing the physical metallurgy events must be superimposed on to the thermomechanical model. Leduc $^{6}$ did this for plain carbon steels using Harding's ${ }^{4)}$ thermal model and assuming homogeneous deformation in each roll gap. Since this early work, the finite difference model has been extended to cover a range of metals and has been applied to several different types of flat rolling process. The computer program now comes under the name SLIMMER: Sheffield Leicester Integrated Model for Microstructural Evolution in Rolling. This paper describes the program in detail and presents examples of its capabilities.

\section{Background Physical Metallurgy}

Much of the metallurgy of hot working is now well understood and can be described in sound physical terms. Nevertheless, the desire to provide models for the evolution of microstructure during thermomechanical processing necessitates that empiricism be used to fill the gaps in understanding. This section presents the formulae used to describe the evolution and outcome of microstructural events, indicating their physical basis and where empiricism is still used.

\subsection{Dynamic Events}

The dynamic events are those microstructural changes occurring during the deformation. For thermomechanical processing the important phenomena are work hardening, dynamic recovery and dynamic recrystallization. The considerable ease of self diffusion at high temperatures ensures that recovery processes are active as soon as dislocations appear during plastic deformation. For metals such as aluminium and ferritic stain- 


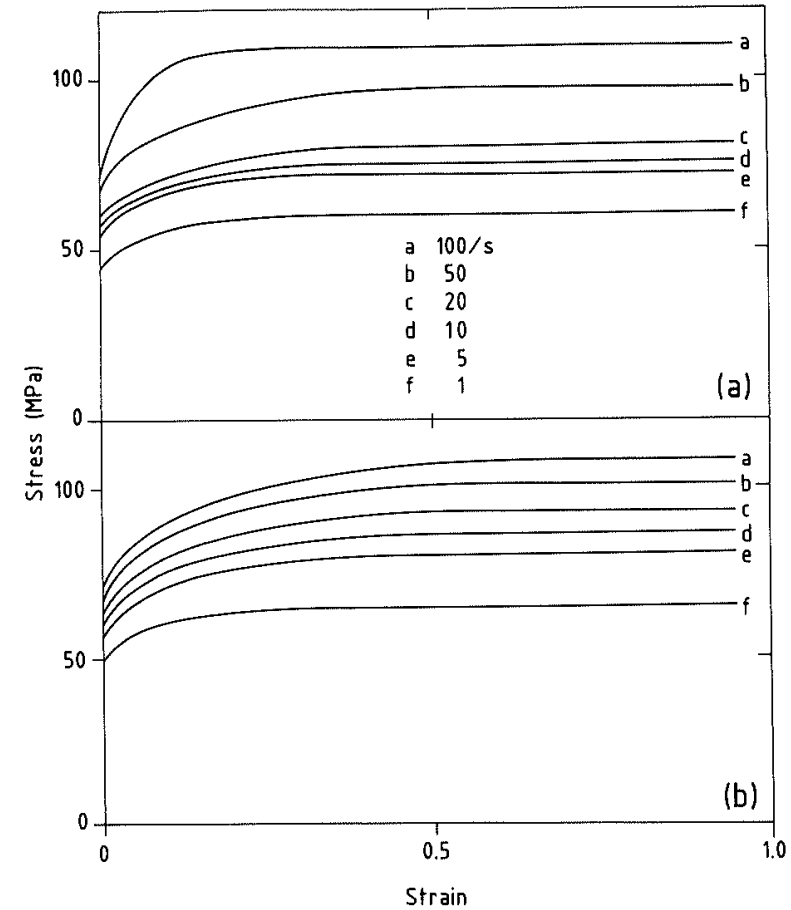

Fig. 1. (a) Stress-strain curves for $\mathrm{Al}-1 \% \mathrm{Mg}$ measured using plane strain compression testing ${ }^{21)}$ at $400^{\circ} \mathrm{C}$ and different strain rates;

(b) Computed stress-strain curves for $\mathrm{Al}-1 \% \mathrm{Mg}$ for same conditions as (a).

less steels, once recovery is established, it is sufficiently efficient to counteract any further work hardening and the stress-strain curve becomes horizontal, Fig. 1. The predominant microstructural feature of dynamically recovering metals is a well-defined subgrain structure. The level of flow stress depends on the prevailing conditions of temperature, strain and strain rate. The stress-strain equation used in SLIMMER for aluminium and $\mathrm{Al}-1 \% \mathrm{Mg}$ alloy is a generalized Voce type:

$$
\sigma_{e}=\sigma_{o}+\left(\sigma_{s s}-\sigma_{o}\right)[1-\exp (-C \varepsilon)]^{m}
$$

where $\sigma_{e}$ represents the flow stress at a strain of $\varepsilon, \sigma_{o}$ is the stress at a plastic strain of zero, $\sigma_{s s}$ is the steady-state flow stress achieved at larger strains, and $m$ is the work hardening exponent. $C$ is dependent on the deformation conditions, as are $\sigma_{o}$ and $\sigma_{s s}$; the general form for the flow stresses is illustrated by:

$$
\sigma_{o}=A_{1} \sinh ^{-1}\left[\left(\frac{Z}{A_{2}}\right)^{A_{3}}\right]
$$

where $A_{n}$ are material dependent parameters which are measured, and $Z$ is the Zener Hollomon parameter:

$$
Z=\dot{\varepsilon} \exp \left(\frac{Q_{D E F}}{R T_{D E F}}\right)
$$

where $\dot{\varepsilon}$ is strain rate, $Q_{D E F}$ is the apparent activation energy for deformation, $R$ is the Gas constant (8.31 J/K mol) and $T_{D E F}$ is the absolute temperature of the deformation. Figure 1 includes computed curves to compare with the measured ones.

With metals which have less efficient recovery processes, the build-up of dislocations can lead to the

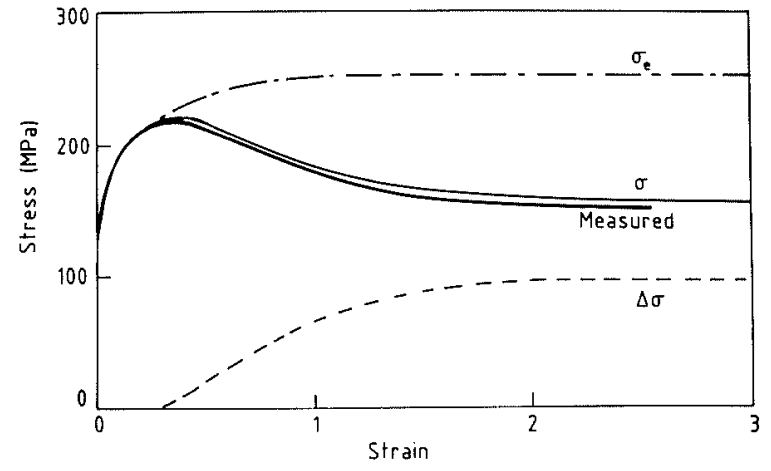

Fig. 2. Illustration of computed and measured stress-strain curve for $\mathrm{C}-\mathrm{Mn}$ steel $^{6\}}$ which displays dynamic recrystallization. Conditions: strain rate $=4.5 / \mathrm{s}$, temperature $=900^{\circ} \mathrm{C}$, initial grain size $=17 \mu \mathrm{m}$.

nucleation and growth of recrystallized grains during the deformation. The appearance of initially unworked crystals produces a drop in the stress-strain curve, but it soon levels off as repeated cycles of work hardening and recrystallization become established. This is dealt with in SLIMMER by subtracting a softening contribution from the flow stress of Eq. (1):

$$
\begin{aligned}
& \Delta \sigma=\left(\sigma_{s s}-\sigma_{s s}^{\prime}\right)\left\{1-\exp \left[-k^{\prime}\left(\frac{\varepsilon-a \varepsilon_{p}}{\varepsilon_{p}}\right)^{m^{\prime}}\right]\right. \\
& \text { for } \varepsilon>a \varepsilon_{p} \ldots . . \\
& \Delta \sigma=0 \text { for } \varepsilon<a \varepsilon_{p}
\end{aligned}
$$

where $\sigma_{s s}^{\prime}$ is the actual steady-state flow stress achieved at larger strains and can be expressed by an equation of type (2). $\varepsilon_{p}$ is the strain for the peak in the flow stress given by:

$$
\varepsilon_{p}=A_{4} d_{o}^{p} Z^{q}
$$

where $d_{o}$ is the grain size prior to the deformation. The parameters $k^{\prime}, a, m^{\prime}, A_{4}, p$ and $q$ are all material dependent.

Figure 2 gives an example of the use of these formulae to construct a stress-strain curve which agrees well with the measured curve over the whole stratin range. Equation (4) is of the Avrami type commonly used to represent recrystallization kinetics. The $m^{\prime}$ value of 1.4 agrees reasonably well with the Avrami coefficients obtained for dynamic recrystallization from metallographic measurements on nickel, ${ }^{7,8)}$ and stainless steel. ${ }^{9)}$

Thus modelling the dynamic microstructural events is important for determining flow stress levels, and hence rolling loads. The second important outcome is that subsequent static (i.e. without concurrent deformation) restoration by recovery or recrystallization is dependent on the microstructure emerging from the deformation. These static events will be dealt with next.

\subsection{Static Events}

Following deformation, even after steady state dynamic recovery or recrystallization has been established, there is still scope for further microstructural change. Such change is driven by the energy stored from the deformation in dislocations and is facilitated by relatively easy diffusion at these high temperatures. 


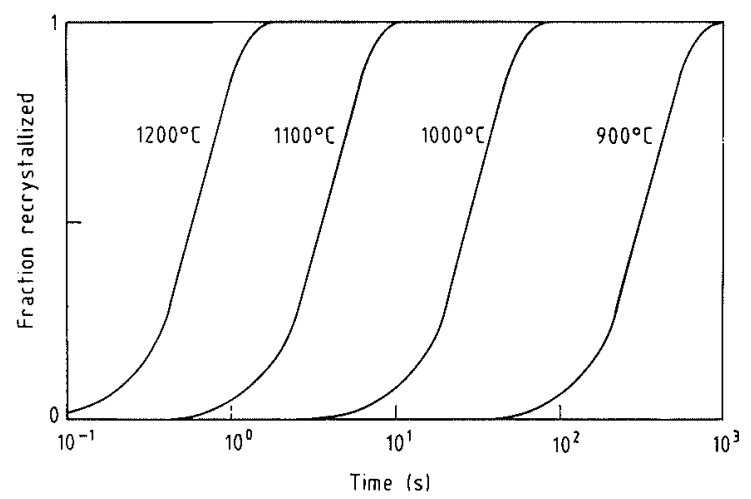

Fig. 3. Kinetics of static recrystallization following an Avrami relationship (Eq. (6)) for $\mathrm{C}-\mathrm{Mn}$ steel previously deformed to a strain of 0.2 at a strain rate of $5 / \mathrm{s}$.

Some static recovery will occur immediately on unloading. Given sufficent time, this will be followed by static recrystallization and, if that is complete, by grain growth. The times needed for these processes vary with alloy and thermomechanical conditions. It is common for recrystallization not to start between stands in a rolling mill, and it is equally common to observe considerable grain growth between stands. Thus the kinetics of these events, as well as the resulting grain size, have to be known accurately for modelling multipass rolling.

The kinetics of static recrystallization in hot working can be well described by the Avrami equation:

$$
X=1-\exp \left[-0.693\left(t / t_{0.5}\right)^{k}\right]
$$

where $X$ is the volume fraction recrystallized after time $t$. The exponent $k$, typically 1 to 2 , is not very sensitive to deformation parameters, whereas the time for half recrystallized, $t_{0.5}$, changes by orders of magnitude over the range of conditions encountered during hot rolling, see example curves in Fig. 3. It is therefore essential to have a quantitative description of $t_{0.5}$, in terms of process variables. Apart from the temperature dependencies arising from the thermally activiated processes, theory does not yet define the form of relationship for other variables, and physically reasonable empirical functions have thus been adopted to describe experimental observations. For example, for C-Mn steels (time in seconds, $d_{o}$ in $\left.\mu \mathrm{m}\right)$ :

$$
\begin{array}{r}
t_{0.5}=2.5 \cdot 10^{-19} d_{o}^{2} \varepsilon^{-4} \exp (300000 / R T) \\
\text { for } \varepsilon<0.8 \varepsilon_{p}
\end{array}
$$

the conditional range implies that dynamic recrystallization had not occurred in the previous deformation. The grain size produced by complete recrystallization without previous dynamic recrystallization, $d_{\text {rex }}$, is described by ${ }^{6)}$

$$
d_{\text {rex }}=0.743 d_{o}^{0.67} \varepsilon^{-1}
$$

Figure 4 illustrates the progress of recrystallizing grains, $d_{r}$, towards $d_{\text {rex }}$, followed by the slower progress of grain growth (see below). As can be seen, the size of the original, unrecrystallized grains, $d_{u}$, falls as they are consumed by new grains. The resulting mean grain size is given by ${ }^{12)}$ :

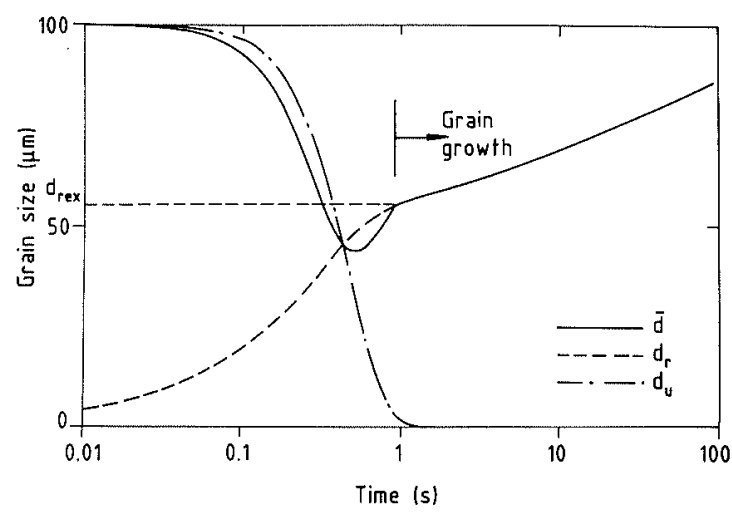

Fig. 4. Grain size evolution through recrystallization and grain growth predicted using Eqs. (8), (9) and (11) for $\mathrm{C}-\mathrm{Mn}$ steel at $1100^{\circ} \mathrm{C}$ previously deformed to a strain of 0.2 at a strain rate of $5 / \mathrm{s} ; \vec{d}=$ mean grain size; $d_{r}=$ recrystallizing grain size; $d_{u}=$ size of original, unrecrystallized, grains; $d_{r e x}=$ fully recrystallized grain size.

$$
\bar{d}=X^{4 / 3} d_{r e x}+(1-X)^{2} d_{o}
$$

Although this equation is used in SLIMMER to calculate $\bar{d}$ during recrystallization, the recrystallized and unrecrystallized grains are assumed to be acting independently. This rather unlikely assumption performs well for modelling aluminium alloys but less so for steels. ${ }^{13)}$ However, the rapid progress of recrystallization in $\mathrm{C}-\mathrm{Mn}$ steels renders the form of dependency of $\bar{d}$ to be of little consequence.

It is dangerous to read too much into the form of empirical equations, but there is compatibility between Eqs. (7) and (8) if the simplest case of site saturation and no grain coarsening during recrystallization is considered. For this, if uniform tetrakaidecahedral grains are assumed, the number of nuclei $N_{v}$ per unit volume is:

$$
N_{v}=0.43 d_{r e x}^{-3}
$$

Since the time for recrystallization is inversely proportional to the nucleation density, the $d_{o}^{2}$ dependence of Eq. (7) can be seen to stem directly from the effect of $d_{o}$ on the density of nucleation sites. A dependence on $d_{0}^{2}$ would arise directly if grain edges provided the only preferential nucleation sites, but in practice it probably reflects involvement also of grain corners and grain interfaces.

Equations (7) and (8) only apply to previously accumulated strains less than the critical strain for the onset (or for the occurrence of some small fraction) of dynamic recrystallization. At this strain, a necklace of small recrystallized grains would have formed around the original grain boundaries during the previous deformation, consuming the most favourable sites for nucleation of static recrystallization. However, some nuclei would be present from the dynamically recrystallized grains which would still be growing when deformation ceased. This leads to a lower limit in the time to the start of static (or metadynamic) recrystallization:

In some alloys precipitation events are particularly important; e.g. niobium carbonitride precipitation, which can stop static recrystallization during hot rolling of 
nobium microalloyed steels. ${ }^{14,15)}$ Such precipitation is accelerated by deformation, hence the thermomechanical history of the steel must be followed to be able to determine the onset of precipitation. Such precipitation will so slow down recrystallization that it can be considered to have stopped within practical rolling times. Figure 5 illustrates the interaction of $\mathrm{Nb}(\mathrm{C}, \mathrm{N})$ precipitation and static recrystallization under isothermal conditions. The three different prior deformations show how sensitive the interaction is to the processing conditions. A single "recrystallization stop temperature" is not adequate to encompass the subtle interaction of precipitation and recrystallization. ${ }^{16)}$

When recrystallization is complete, further growth may take place even in the relatively short times available between passes in hot rolling. The time dependence of grain growth may be represented by the theoretically expected form of equation:

$$
d^{n}=d_{r e x}^{n}+A_{5} t \exp \left(-Q_{g g} / R T\right)
$$

The measured exponent $n$ is commonly higher than the expected value of 2 : a value of 10 is found for $\mathrm{C}-\mathrm{Mn}$ steels. Furthermore, the apparent activation energy for

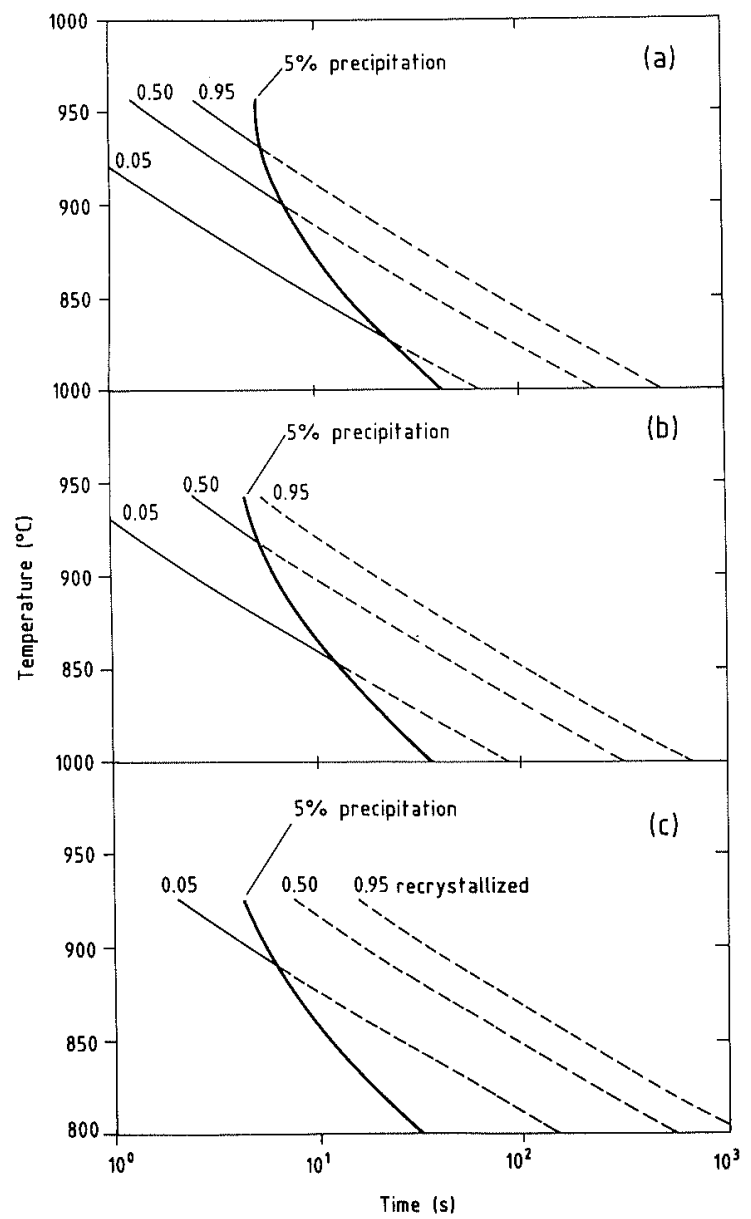

Fig. 5. Interaction of strain induced precipitation and static recrystallization under isothermal holding conditions:

(a) prior strain, $\varepsilon=0.22$ and strain rate, $\dot{\varepsilon}=16.8 / \mathrm{s}$ at a deformation temperature, $T_{D E F}=980^{\circ} \mathrm{C}$ (cf. pass 12 of schedule in Fig. 8);

(b) $\varepsilon=0.20, \varepsilon=17.4 / \mathrm{s}, T_{D E F}=962^{\circ} \mathrm{C}$ (cf. pass 13 in Fig. 8);

(c) $\varepsilon=0.17, \varepsilon=18.1 / \mathrm{s}, T_{D E F}=940^{\circ} \mathrm{C}$ (cf. pass 14 in Fig. 8). grain growth, $Q_{g g}$, may be much higher than expected for diffusion controlled boundary migration. The discrepancies almost certainly arise because the preexponential term, $A_{5}$, which is assumed to be constant in simple theory, is in practice both time and temperature dependent at short growth times, as a result of segregation effects on boundary mobility. Further basic research is required before more sophisticated forms of equation can be justified.

\section{SLIMMER}

SLIMMER couples a thermal and a microstructure model with Sims' hot rolling theory for calculating operating loads. These three aspects will be described separately with their interaction indicated as appropriate.

\subsection{Thermal Model}

At the heart of SLIMMER is a thermal model which uses an explicit finite difference technique to calculate the temperature profile on a transverse section of the stock as it progresses down the hot rolling mille). The assumption of symmetry allows just one quarter of the transverse section to be modelled. The initial temperature of the stock can be input as a uniform single value or as a profile, perhaps from a calculation made by another program for a reheating furnace.

The section is subdivided into quadrilateral elements whose future temperatures are calculated in terms of the current temperatures of each element and its neighbours. Correct calculation of the time step for this prediction is essential for stability of the solution and is determined automatically by the program for each period of air, water or roll cooling down the mill.

The thermal influences on the stock are shown schematically in Fig. 6. Between stands heat is lost by convection and radiation to the air, and more rapidly during descaling or cooling by water. Contact with the roll, under load, provides an efficient conduction path to a heat sink which results in sudden temperature drop of the surface of the stock. However, on emerging from the roll stand the surface quickly recalesces by heat flowing from the centre of the stock.

For the interstand cooling, heat loss is expressed in a

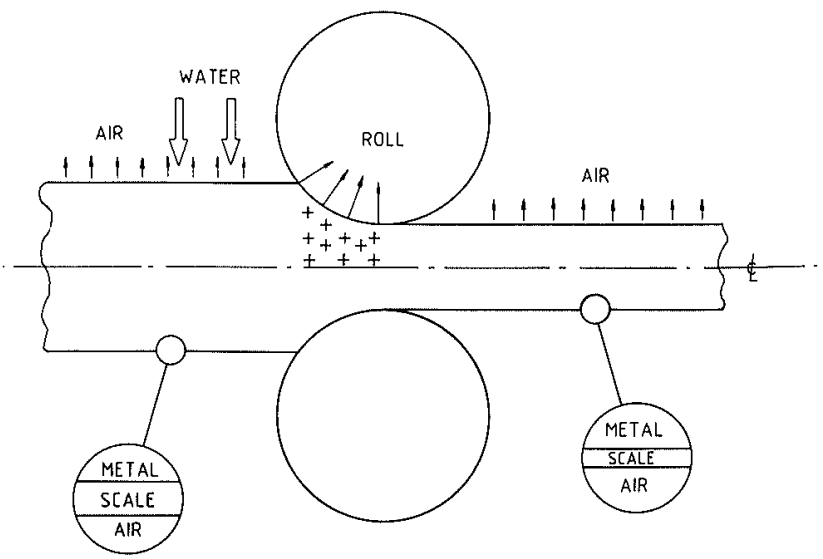

Fig. 6. Schematic illustration of heat sinks and source in hot rolling; + indicates heat generation due to deformation, thin arrows indicate direction of heat flow. 
standard manner:

$$
Q_{1}=\lambda \sigma\left(T_{s}^{4}-T_{\infty}^{4}\right)+H_{c}\left(T_{s}-T_{\infty}\right)^{n}
$$

where $T_{s}$ is the surface temperature of the stock and $T_{\infty}$ that of the ambient; $\sigma$ is the Stefan Boltzmann constant $\left(5.67 \times 10^{-8} \mathrm{~W} / \mathrm{m}^{2} \mathrm{~K}^{4}\right)$. The values of emissivity $\lambda$ and convection coefficient $H_{c}$ have been determined by experiment. The exponent $n$ is $5 / 4$ for natural convection, but different for other circumstances.

Heat loss to the roll is by conduction only:

$$
Q_{2}=H_{R}\left(T_{S}-T_{R}\right)
$$

where $T_{R}$ is the surface temperature of the roll and the coefficient $H_{R}$ must be determined by experiment. The temperature profile within the roll is calculated, although the computation time can be reduced by using the "phantom roll technique". This technique exploits the essential symmetry of temperature profiles in the stock and roll and uses their thermal properties to obviate the need to calculate a full roll temperature profile while retaining the heat sink characteristics of the roll. ${ }^{18)}$

Oxidation of the stock alters the efficiency of heat loss to the atmosphere and this is accounted for by recalculating the surface temperature as that of the oxide rather than the stock. Each rolling reduction will also thin this oxide layer as indicated in Fig. 6. Correct estimation of the oxide layer is important for calculating heat loss both during rolling and between stands, particularly for accelerated water cooling. ${ }^{19)}$

Heat flow within the stock is by conduction and for its calculation the program uses temperature dependent physical properties, viz, thermal conductivity, specific heat and density. During a roll pass, most of the mechanical energy is transformed into heat. This deformation heating is determined for each element at each time step using a mean flow stress obtained by integrating Eq. (1), supplemented by Eq. (4) for steels. Heat generated by friction is neglected as insignificant: this is supported by others. ${ }^{20)}$

\subsection{Microstructure Model}

The sensitivity of the microstructural events, described in Sec. 2, to the prevailing temperature of the stock make it essential that an accurate and detailed distribution of temperature is calculated. The previous section describes how this is done in SLIMMER. The other determining operating variables in the microstructural changes are strain and strain rate. In SLIMMER these are assumed to be homogeneously distributed through the thickness and across the width of the stock and are calculated as:

$$
\begin{gathered}
\varepsilon=\frac{2}{\sqrt{3}} \ln \frac{h_{o}}{h_{f}} . . \\
\dot{\varepsilon}=\frac{\varepsilon V_{R}}{\sqrt{R\left(h_{o}-h_{f}\right)}}
\end{gathered}
$$

where $h_{o}$ and $h_{f}$ are the entry and exit thicknesses of the stock, respectively, $V_{R}$ is the peripheral roll speed, and $R$ is the roll radius.

When modelling the hot rolling of aluminium, the roughing passes involve rolling stock which is very thick compared to the roll radius. This will produce a distribution of deformation which is far from being uniform through the stock thickness. As a first approximation solution to this problem, a parabolic distribution of strain is imposed increasing from the nominal value of Eq. (14) at the centre according to:

$$
\varepsilon_{x}=\varepsilon\left[1+1.76\left(\frac{h_{f}}{R}\right)^{0.5}\left(\frac{x}{h_{f}}\right)^{2}\right]
$$

where $\varepsilon_{x}$ is the strain at a fractional distance $\left(x / h_{f}\right)$ from the centre of the stock of final thickness $h_{f}$. It should be noted that this is an empirical equation whose contribution decreases as roll radius, $R$, increases and as stock thickness decreases. ${ }^{21)}$

This rather crude attempt to account for heterogeneity of deformation is being replaced by the introduction of the finite element method to the roll gap calculations. ${ }^{12,22,23)}$ However, the examples presented in Sec. 4 did not use this version.

Thus a complete thermomechanical history of the stock is determined and the formulae given in Sec. 2 can be used to follow the evolution of microstructure down the rolling mill. However, these formulae imply that the microstructural events occur isothermally. This is clearly inappropriate for an industrial rolling schedule. Provided that the various activation energies do not vary with temperature, allowance can readily be made for non-isothermal interstand holding. This is done by defining a temperature compensated time:

$$
w=\sum_{i} \delta t_{i} \exp \left(-Q / R T_{i}\right)
$$

where $\delta t_{i}$ is the finite difference time step at which the temperature was $T_{i}$; $Q$ is the activation energy of the process for which the calculation is being made. $\omega$ can then be used in a modified Avrami equation (cf. Eq. (6)) for recrystallization:

$$
X=1-\exp \left[-0.693\left(w / w_{0.5}\right)^{k}\right]
$$

Processes with different forms of kinetics, such as that for grain growth (Eq. (11)), are treated in a similar manner.

One complication is the need to account for partially recrystallized regions receiving a further deformation. The approach taken in SLIMMER is to subdivide the region according to the amount of recrystallization and to follow each new sub-region separately for the remainder of the rolling process. Clearly, several cycles of partial recrystallization within these sub-regions result in a plethora of ever smaller zones with different microstructures. To prevent these zones becoming insignificantly small, a limit of $5 \%$ recrystallization must be exceeded before a new sub-region is created. Likewise, in excess of $95 \%$ recrystallization is rounded up to $100 \%$. Figure 7 illustrates this accounting procedure by means of a flow chart.

\subsection{Operating Loads}

The equations for flow stress presented in Sec. 2 allow the mean flow stress for the stock during a rolling pass to be calculated with a reasonable degree of accuracy. 


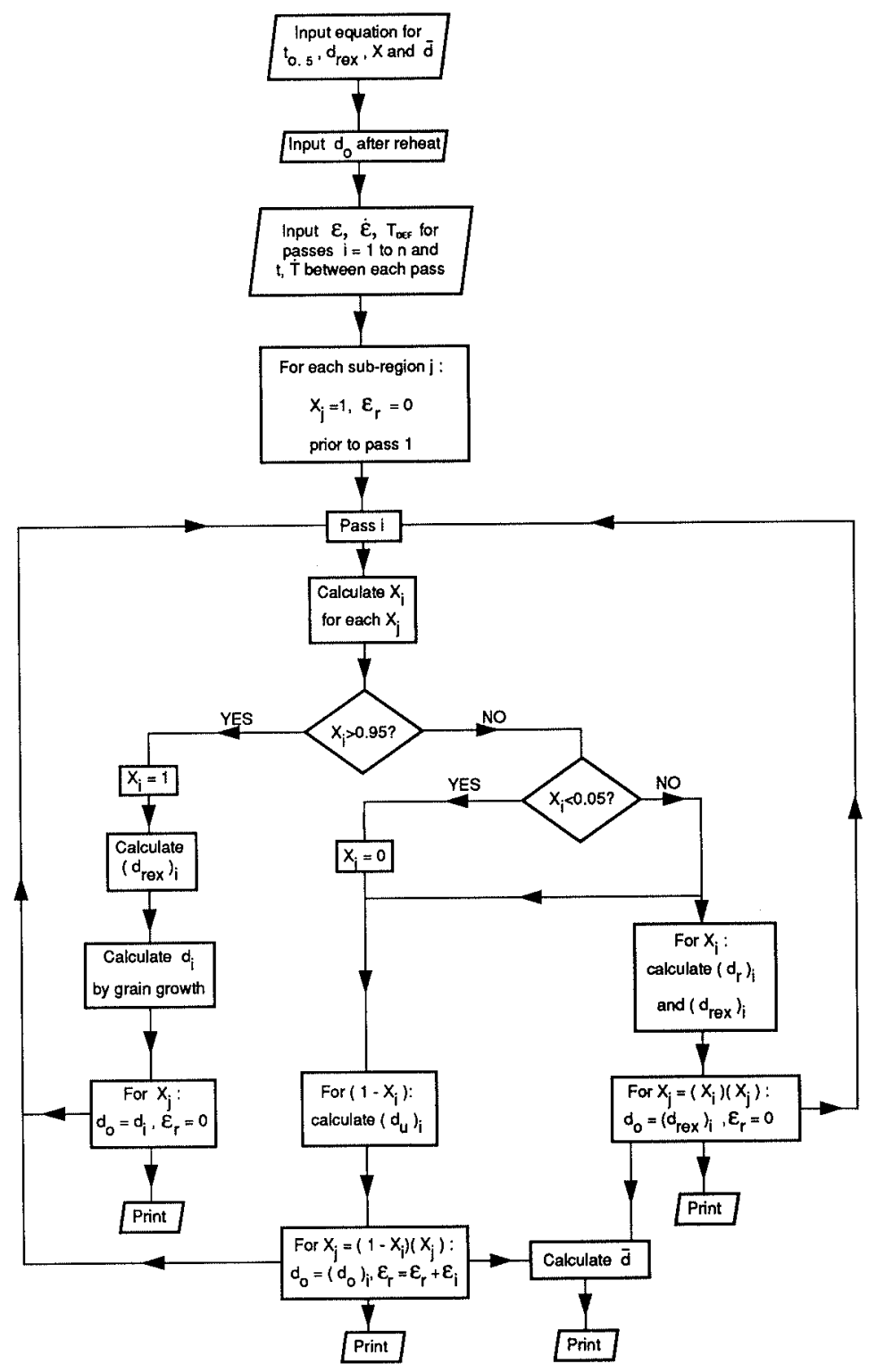

Fig. 7.

Flow chart indicating procedure for following microstructure evolution in SLIMMER. $\varepsilon_{r}$ is strain retained in unrecrystallized region prior to a pass; other symbols as in text.
Sims $^{24)}$ theory for hot rolling is used to compute the rolling load and torque. This theory assumes sticking friction and uniform, through-thickness deformation. However, deriving the flow stress from a detailed knowledge of the temperature distribution and sound, experimentally-based flow stress formulae allows a good estimate of the operating forces to be made.

\subsection{Running SLIMMER}

SLIMMER began as a mainframe FORTRAN program, but improved computer power at lower cost means that it now runs on a PC. A Windows "C" version in currently being prepared.

Table 1 summarises the input data required to run SLIMMER. Generic metal types have been studied to provide materials data. Other input data are confined to schedule details. Internal variables required by SLIMMER, such a time steps and subdivision of the section into elements, are either calculated automatically within SLIMMER or reasonable default values are provided which can be modified by the sophisticated user.

The standard output of SLIMMER is graphical displays of temperature and grain size evolution down the
Table 1. Input data for SLIMMER.

\begin{tabular}{ll}
\hline Metal code & $\begin{array}{l}\text { to access appropriate microstructural equa- } \\
\text { tions and physical properties; currently } \\
\text { available are aluminium, aluminium } 1 \% \\
\text { magnesium, carbon-manganese steel, niobi- } \\
\text { um microalloyed steel, 304 stainless steel and } \\
\\
316 \text { stainless steel }\end{array}$ \\
Initial stock conditions & $\begin{array}{l}\text { thickness, width, oxide thickness (steels } \\
\text { only), grain size, reheat temperature (uni- } \\
\text { form or full section profile) } \\
\text { number and timing of rolling passes, } \\
\text { descalers, water cooling } \\
\text { reduction, spread, roll radius, roll speed, } \\
\text { initial roll temperature }\end{array}$
\end{tabular}

rolling mill, supplemented by a detailed table of calculated values. The next section gives examples of such output, including comparison with measurements. Other examples can be found in the literature. ${ }^{13,17,19,21,25,26)}$

\section{Applications of SLIMMER}

SLIMMER is a process model designed for use off-line to assist with schedule development, design or redesign 
of mill configurations, problem investigation, and as a general purpose "what if" tool. This section presents two examples to illustrate the capabilities of the program and to show some of the validation of the predictions.

\subsection{Rolling Nb-microalloyed Steel Plate}

A plate rolling schedule has been modelled for which

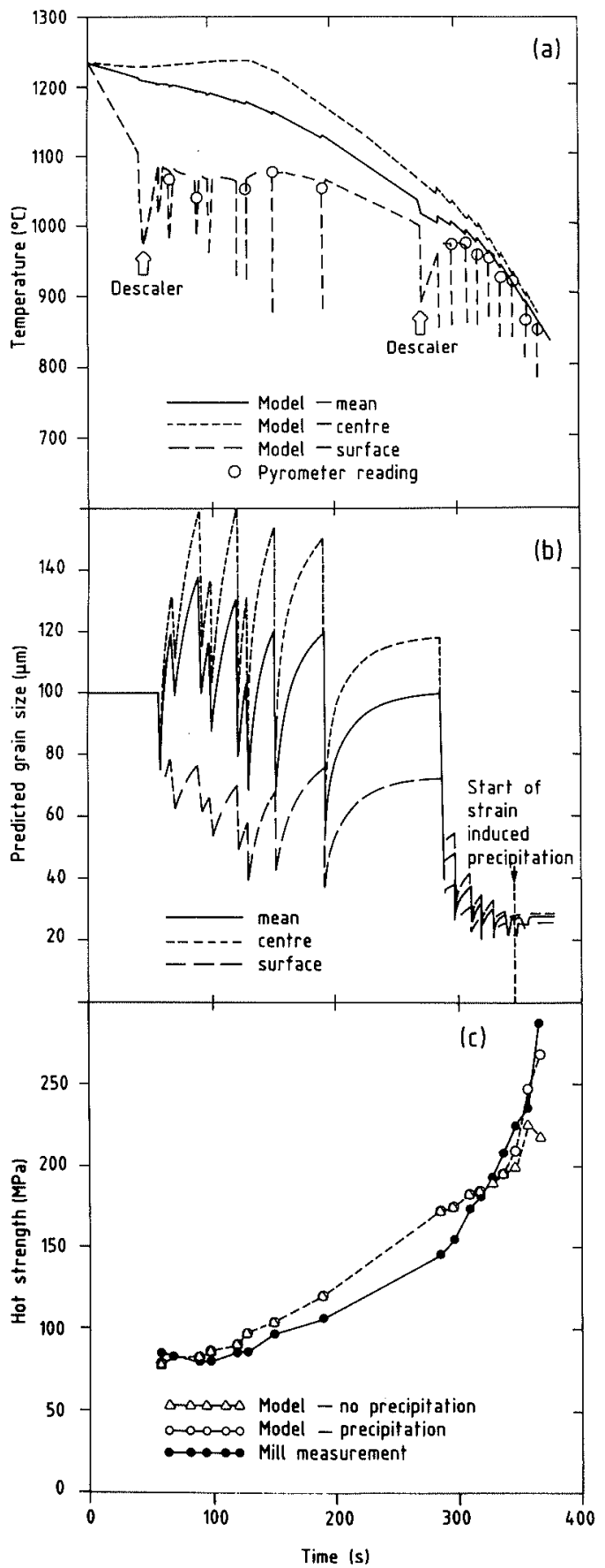

Fig. 8. SLIMMER predictions for an industrial plate rolling schedule with niobium microalloyed steel for an overall reduction from 230 to $11 \mathrm{~mm}$.

(a) Temperature predictions averaged across the centre and surface rows together with the section mean. Pyrometer readings are indicated.

(b) Grain sizes computed for centre, surface and mean layers.

(c) Hot strength derived from roll force calculations for strain induced precipitation stopping or not affecting static recrystallization in comparison with strength values derived from mill data. measured pyrometer temperature readings and rolling loads for niobium-microalloyed steel were available. The chemical composition of the steel was (wt\%): $0.145 \mathrm{C}$, $0.0045 \mathrm{~N}, 0.021 \mathrm{Nb}, 1.20 \mathrm{Mn}, 0.21 \mathrm{Si}, 0.023 \mathrm{P}$ and $0.010 \mathrm{~S}$. The grain size prior to the first roughing pass was guessed to be $100 \mu \mathrm{m}$ : the multiple cycles of deformation and recrystallization which follow mean that the accuracy of this guess is not important for the grain sizes in the schedule (see Sec. 4.2).

Figure 8(a) shows the temperatures calculated by SLIMMER together with recorded pyrometer readings. The predicted surface temperature profile matches well with these measurements.

Figure 8(b) shows the predicted microstructure evolution during the schedule. The temperature gradients in Fig. 8(a) result in different recrystallization kinetics across the section. The grain growth is particularly significant after the eight roughing passes and illustrates the need for reliable expressions for the kinetics of grain growth. For Nb-microalloyed steels the following equation is used ${ }^{27)}$ (cf. Eq. (11)):

$$
d^{4.5}=d_{r e x}^{4.5}+4.05 \cdot 10^{23} t \exp (-435000 / R T)
$$

As the plate becomes thinner, the temperature and microstructure gradients through the thickness of the stock diminish. The start of strain induced precipitation is indicated as taking place between passes 14 and 15 where recrystallization was stopped in the outer layers of the plate. Precipitation was initiated (effectively $5 \%$ ) in the remaining layers before pass 16 , thereby completing the stopping of static recrystallization throughout the plate. Using a critical strain of $0.7 \varepsilon_{p}$ for its onset did not trigger dynamic recrystallization for any of the passes in this schedule.

Figure 8(c) shows the computed mean flow stress for each pass in comparison with that derived from the measured mill forces. The match is reasonable throughout the schedule, notable for the last few passes where strain induced precipitation was predicted. Stopping static recrystallization results in increased work hardening and a larger mean flow stress for the pass. Modelling without precipitation stopping recrystallization results in a markedly lower flow stress.

The use of temperature compensated time to account properly for non-isothermal temperature histories is an accurate way of determining the onset, in this case, of strain induced precipitation. It also allows this onset to be determined for different layers through the thickness of the stock. The conditions selected for Fig. 5 were those at the exit of passes 12,13 and 14 of this example schedule, the temperature of deformation being the average value for the section. The plots in Fig. 5 are isothermal curves and can be used to give an indication of how much metal recrystallized before precipitation effectively stopped the process at any given holding temperature. However, it is a relatively clumsy means of assessing these events and cannot have the precision of the method employed in SLIMMER.

\subsection{Effect of Initial Grain Size}

For C-Mn steel under practical hot rolling conditions, 


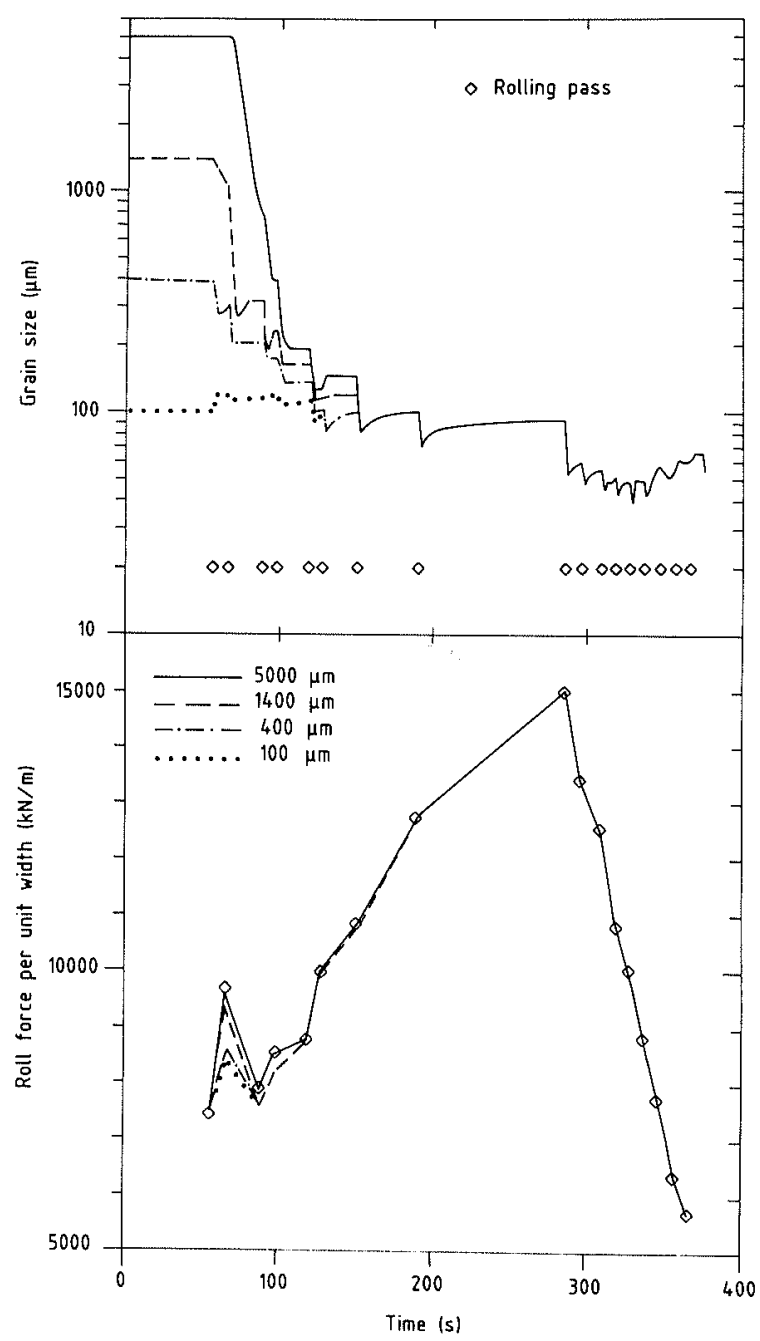

Fig. 9. SLIMMER predictions for the same industrial plate schedule in Fig. 8 but with $\mathrm{C}-\mathrm{Mn}$ steel.

(a) Stock centre grain sizes are shown for four computations which differed only in the starting grain size.

(b) Resulting roll force calculations where differences are due to different amounts of interstand static recrystallization altering the amount of work hardening.

recrystallization always leads to significant grain refinement-in contrast to the behaviour of aluminium alloys. This is dramatically illustrated in Fig. 9(a) where the same plate schedule of Fig. 8 has been used for C-Mn steel of widely varying initial grain sizes. The largest grain size could result from extended reheating of continuously cast stock. However, by the end of roughing, several cycles of recrystallization have produced a convergence of grain size. Thus, provided there are sufficient cycles of recrystallization, modelling $\mathrm{C}-\mathrm{Mn}$ steels does not require an accurate measure of initial grain size. It should be noted that the contrasting tendency of aluminium and its alloys to recrystallize to larger grains means that a detailed knowledge of the initial state is critical.

One interesting consequence of very large initial grain size is its effect on the kinetics of static recrystallization, Eq. (7). Figure 9(a) shows that for $d_{o}=5000 \mu \mathrm{m}$, no static recrystallization takes place between passes 1 and 2 compared with full recrystallization for $d_{o}=100 \mu \mathrm{m}$, with
400 and $1400 \mu \mathrm{m}$ being 90 and $21 \%$ recrystallized, respectively. Incomplete recrystallization results in a higher flow stress during the next rolling pass due to work hardening; this can be seen in Fig. 9(b), most notably for pass 2 where the rolling load for the $5000 \mu \mathrm{m}$ stock is $13 \%$ higher than for the $100 \mu \mathrm{m}$ stock. This would need to be counteracted by applying more strain in the first pass to accelerate the kinetics of static recrystallization (see Eq. (7)) and possibly by increasing the interpass time between passes 1 and 2 .

\section{Conclusions}

Although empirical, the equations used to describe the evolution of microstructure are physically reasonable. Despite the assumptions which are made, the predictions computed by SLIMMER generally accord with experience. Where measured data are available they agree quantitatively with the predictions. Examples presented which show the effect of microstructure evolution on rolling loads emphasise the importance of microstructure modelling for the mill engineer.

Models such as SLIMMER also play a major role in enhancing understanding of the metallurgy of hot working. The inability of current physical metallurgical theories to cope with the needs of SLIMMER has been a source of direction for fundamental research.

\section{Acknowledgements}

The Science and Engineering Research Council are thanked both for research grants and for the support of some of the many students who have provided ideas and data essential to the development of SLIMMER.

\section{REFERENCES}

1) C. M. Sellars and W. J. McG. Tegart: Mem. Sci. Rev. Met., 63 (1966), 731.

2) J. J. Jonas, C. M. Sellars and W. J. McG. Tegart; Met. Rev., 14 (1969), 1.

3) F. Hollander: Mathematical Models in Metallurgical Process Development, Iron Steel Inst., London, (1970), 46.

4) R. A. Harding: Ph. D. thesis, University of Sheffield, (I976).

5) M. Pietrzyk and J. G. Lenard: Thermal-mechanical Modelling of the Flat Rolling Process, Springer-Verlag, Berlin, (1991).

6) L. A. Leduc: Ph. D. thesis, University of Sheffield, (1980).

7) J. P. Sah, G. J. Richardson and C. M. Sellars: J. Aust. Inst Met., 14 (1969), 292.

8) C. M. Sellars: Metalurgia i Odlevnictvo, 3 (1979), 376.

9) W. Roberts, H. Boden and B. Ahlblom; Met Sci., 13 (1979), 195.

10) D. R. Barraclough and C. M. Sellars: Met. Sci., 13 (1979), 257.

11) G. C. Reyes and J. H. Beynon: Hot Deformation of Aluminium Alloys, T.M.S., Warrendale, (1991), 491.

12) J. H. Beynon, A. R. S. Ponter and C. M. Sellars: Modelling of Metal Forming Processes, Kluwer Acad. Publ., Dordrecht, Netherlands, (1988), 321.

13) C. M. Sellars; Mat. Sci. Tech., 6 (1990), 1072.

14) B. Dutta and C. M. Sellars: Mat. Sci. Tech., 3 (1987), 197.

15) E. Valdes and C. M. Sellars: Mat. Sci. Tech., 7 (1991), 622.

16) J. J. Jonas and C. M. Sellars: Aspects of the Future Development of Metals and Ceramics, to be published by Inst. Met., (1991).

17) C. M. Sellars: Hot Working and Forming Processes, Met. Soc., London, (1980), 3.

18) R. Kawai: M. Phil. thesis, University of Sheffield, (1985).

19) R. Colas and C. M. Sellars: Accelerated Cooling of Rolled Steel, Pergamon, New York, (1988), 121.

20) P. D. Hodgson: B. H. P. Melbourne Research Laboratories, 
Australia, private communication, (1990).

21) E. S. Puchi, J. H. Beynon and C. M. Sellars: Thermec-88, ISIJ, Tokyo, (1988), 572.

22) J. H. Beynon, P. R. Brown, S. I. Mizban, A. R. S. Ponter and C. M. Sellars: Numiform '86, Balkema, Rotterdam, (1986), 213.

23) J. H. Beynon, P. R. Brown, S. I. Mizban, A. R. S. Ponter and C. M. Sellars: Computational Methods for Predicting Material Processing Defects, Elsevier, Amsterdam, (1987), 19.
24) R. B. Sims: Proc. Inst. Mech. Engrs., 168 (1954), 191.

25) C. M. Sellars and J. A. Whiteman: Met. Sci., 13 (1979), 187

26) J. H. Beynon: Recrystallization ' 90 , T.M.S., Warrendale, (1990), 429.

27) P. D. Hodgson and R. K. Gibbs: Modelling of Metal Rolling Processes-Symp. 2: Control of Properties in Hot Rolling, Inst. Met., London, 13 June 1991, Paper 4. 